\title{
Economic Evaluation of the Use of Cefotaxime and Ceftazidime in the Treatment of Pneumonia in Pediatric Patients
}

\author{
Valen Ruterlin ${ }^{1,2}$, Rano K. Sinuraya ${ }^{2}$, Eli Halimah², Melisa I. Barliana ${ }^{3}$, Sri Hartini ${ }^{4}$ \\ ${ }^{1}$ Anutapura Hospital, Palu, Central Sulawesi, Indonesia \\ ${ }^{2}$ Department of Pharmacology and Clinical Pharmacy, Faculty of Pharmacy, Universitas Padjadjaran, \\ Jatinangor, West Java, Indonesia \\ ${ }^{3}$ Department of Biology Pharmacy, Faculty of Pharmacy, Universitas Padjadjaran, Jatinangor, West \\ Java, Indonesia
}

\begin{abstract}
The prevalence of pneumonia is particularly high among pediatric patients. Appropriate antibiotics selection is required to reduce mortality and morbidity rates associated with these diseases. However, information on cost-effectiveness of empirical antibiotics treatment for pneumonia was limited. This study was aimed to evaluate cost-effectiveness of cefotaxime and ceftazidime for pneumonia in pediatric patients. This study was a retrospective cross sectional study conducted at a hospital in Bandung during January-December 2012. Data were derived from medical records of pediatric pneumonia inpatients during study period. Cost was calculated based on direct medical cost, i.e., inpatient care, medical support, and medicines that were used from admission until hospital discharge. The results showed that there was no statistical difference in the average medical cost of the treatment using cefotaxime (1,197,017 IDR) and ceftazidime (2,245,748 IDR). Incremental cost effectiveness ratio (ICER) showed that cefotaxime is more cost effective than ceftazidime with greater reduction of leukocytes level $\left(576 \mathrm{IDR} / \mathrm{mm}^{3}\right)$. The use of cefotaxime is recommended for the treatment of pnuemonia in pediatric patients.
\end{abstract}

Keywords: cost minimization, cost effectiveness, cefotaxime, ceftazidime, pneumonia

\section{Introduction}

Pneumonia is one of the leading cause of worldwide morbidity and mortality among children. Pneumonia causes deaths in nearly one million children under 5 years of age, annualy. Pneumonia symptoms are nonspecific in younger infants, but cough and tachypnea are usually present in older children. Etiological causative agents of pneumonia are bacteria, viruses, parasites, and fungi. For children aged three months to five years, Streptococcus pneumoniae (S. pneumonie) has been the most frequent bacterial organism causing this devastating disease. ${ }^{1,2}$

Empiric antibiotic treatment remain one of

Corresponding author: Valen Ruterlin, Anutapura Hospital, Palu, Central Sulawesi, Indonesia, Email: valen.ruterlin@gmail.com 
the cornerstone to reduce pneumonia rates. Penicillins and third generation cephalosporins, including cefotaxime and ceftazidime, were proven to be effective even in children with pneumonia due to penicillinresistant $S$ pneumoniae. Third generation of cephalosporins are characterised by a broad spectrum of activity and increased stability to beta-lactamases compared with the first and second generation cephalosporins. ${ }^{3,4}$

The cost of antibiotic treatment is one of the significant medical cost of pneumonia treatment. Ceftazidime is more sensitive compared to cefotaxime, but more expensive. Pharmacoeconomic analysis is required to assess which treatment is more effective, in terms of cost and efficacy. ${ }^{5-7}$ The use of pharmacoeconomic methodology could ensure more realistic cost estimation. The result of this study can be used in the decision-making process to improve health care. $^{8}$

\section{Methods}

In this study, the retrospective cross sectional data were obtained from medical records at a hospital in Bandung, Indonesia, during January-December 2012. The consecutive sampling method was used in this study. The inclusion criteria was pneumonia inpatient children (aged 1-5 years old) who obtained empiric antibiotic therapy, either cefotaxime or ceftazidime. We excluded subjects who did not complete the therapy and had coexisiting diseases. Informed consent was not required as the retrospective study did not change the treatment given to the subjects.

Data source in this study was medical records of the patients. From each participants, data regarding direct medical cost, including inpatient care, laboratory results, and antibiotic treatment were obtained. All of these costs are calculated per day according to the patient's usage and multiplied by the total length of stay. The cost of the drug included antibiotics treatment, parenteral preparations, symptomatic drug treatment, .e.g., ambroxol, dexamethasone, and paracetamol. The total drugs cost were calculated according to the price list of drugs in 2012. Laboratory costs are the supporting costs incurred by the patient during diagnosis, monitoring of adverse events, progress of therapy or determining the outcome of therapy. Laboratory examination for pneumonia patients could include examination of thorax, microbiological profiles, complete blood, and electrolytes. The overall cost is only calculated at the time of examination.

The reduction of leukocytes before and after the treatment was used as parameter of costeffectiveness. Cost minimalization analysis was conducted by comparing the mean of the lowest direct medical cost between two treatment groups. Cost effectiveness analysis was calculated based on the total of antibiotic cost treatment and the effectiveness of antibotics. The data were analyzed using student T-test with 95\% confidence level.

\section{Results and Discussion}

A total of 58 medical record data of subjects were included in this study. We found out that the biggest part of direct medical costs came from inpatient care costs, followed by drugs and laboratory costs. Overall, direct medical cost of treatment with ceftazidime was higher than that of cefotaxime, with the total costs of IDR 2,245,748 and IDR 1,197017 , per inpatient ( 5 days hospital stay), regardless of effectiveness, respectively. No significant differences observed between the costs for these two treatment, which was shown by $p$-value $>0.005$. The results of cost minimization analysis was provided in Table 1. 
Table 1. Cost minimization analysis of cefotaxime dan ceftazidime

\begin{tabular}{lccc}
\hline Direct Medical Cost & Cefotaxime $\mathbf{n = 4 0}$ & Ceftazidime $\mathbf{n = 1 8}$ & P-value \\
\hline Inpatient Care & IDR 734,573 & IDR 1,558,997 & 0.98 \\
Laboratory & IDR 203,886 & IDR 228,492 & 0.87 \\
Drugs & IDR 258,559 & IDR 458,260 & 0.68 \\
\hline Mean & IDR $1,197,017$ & IDR 2,245,748 & 0.64 \\
\hline
\end{tabular}

Average Cost Effectiveness Ratio (ACER) was calculated based on the total cost of antibiotic treatment and the effectiveness of antibiotics. The lower the value of ACER, the more cost effective the treatment. ${ }^{9,10}$ The effectiveness was calculated based on the mean reduction of leukocyte value. ACER in cefotaxime and ceftazidime groups were IDR 20,279 and IDR 29,082, respectively. Incremental analysis showed that using cefotaxime instead of ceftazidime resulted in the saving of IDR $576 / \mathrm{mm}^{3}$ reduction of leukocytes (Table 2). It indicated that the cost of cefotaxime was comparable with its effectiveness ( $3^{\text {rd }}$ quadrant) (Figure 1).

This study assessed the cost minimization and cost effectiveness of two empirical antimicrobial strategies to treat pneumonia in pediatric patients, namely cefotaxime and ceftazidime. The finding showed that treatment with cefotaxime dominated ceftazidime in term of cost and efficacy. In choosing the optimal antibiotic treatment strategy for pneumonia, effectiveness, cost-effectiveness and ecological effects of antibiotics should be taken into account. Optimally, this would consist of a strategy associated with the best patient outcome at the lowest price and with least selective pressure for antibiotic resistance. ${ }^{11}$
Cefotaxime and ceftazidime were the most frequently used antibiotics for pneumonia treatment. The main isolated bacteria in pneumonia patients were S. pneumoniae and $S$. aureus. Most of the microorganisms were sensitive to ceftriaxone, ceftazidime, and cefotaxime, and were resistant to penicillin, ampicillin, and erythromycin..$^{12,13}$

Previous study showed that pneumonia treatment using ceftazidime was succesful in $87 \%$ of the patients, whereas cefotaxime was $77 \%$. Differences in the efficacy may be resulted from prolonged action of cefotaxime, which was recognized to be a drug of choice among $3^{\text {rd }}$ generation of cephalosporin. Our finding is comparable with previous study conducted in Spain, showing that cost effectiveness of cefotaxime is higher than that of other cephalosporins such as ceftriaxone. ${ }^{12-14}$

Limitation of this study included the absent of sensitivity analysis to evaluate which parameters most sensitive to ICER. Besides, this study conducted only in a hospital in Bandung, thus generalizability of this result for Indonesian population is limited.

Table 2. Cost effectiveness analysis of cefotaxime and ceftazidime

\begin{tabular}{lcc}
\hline \multicolumn{1}{c}{ Parameters } & Cefotaxime $\mathbf{n}=\mathbf{1 8}$ & Ceftazidime $\mathbf{n}=\mathbf{4 0}$ \\
\hline Total cost & IDR $1,197,017$ & IDR $2,245,748$ \\
Leucocyte & $5.902 \mathrm{~mm}^{3}$ & $7.722 \mathrm{~mm}^{3}$ \\
ACER & IDR 20,279 & IDR 29,082 \\
ICER & IDR 576,2 & IDR 576,2 \\
\hline
\end{tabular}




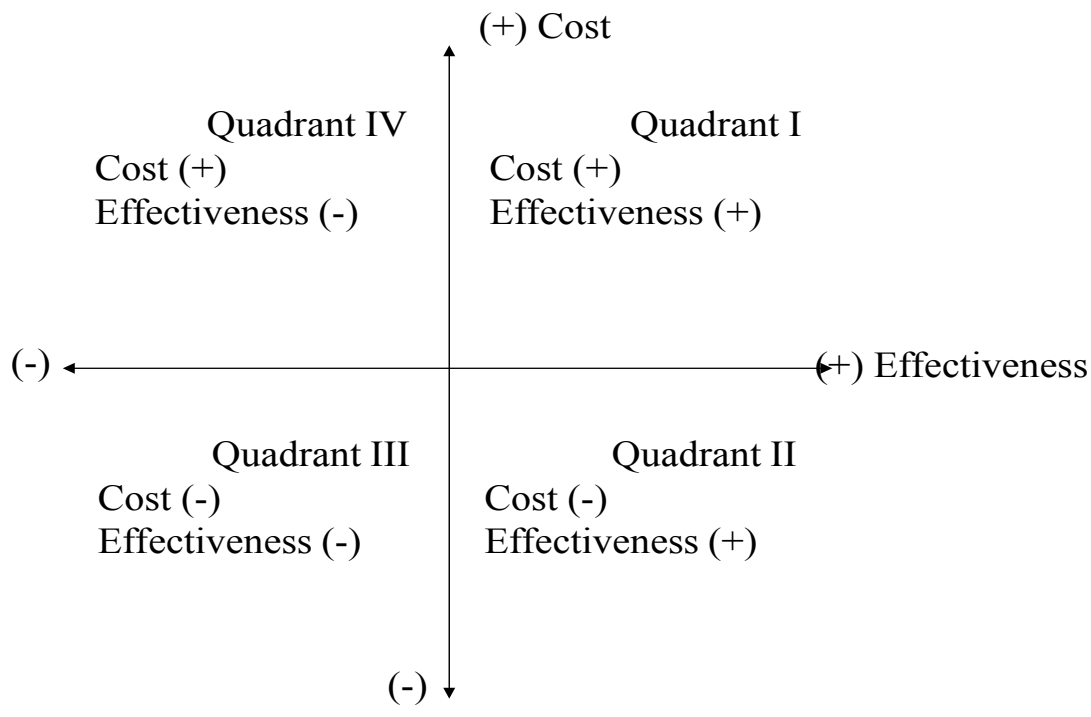

Figure 1. Quadrant of cost effectiveness analysis ${ }^{11}$

\section{Conclusion}

Cefotaxime was more cost effective than ceftazidime, therefore it is recommended for the treatment of pneumonia in pediatric patients.

\section{Acknowledgement}

None declared

\section{Funding}

This study was not funded by any source of grants

\section{Conflict of Interest}

The authors declared no potential conflicts of interest with respect to the research authorship, and/or publication of this article

\section{References}

1. Chetty K, Thomson AH. Management of community-acquired pneumonia in children.Pediatric Drugs. 2007;9(6):401411.

2. Konomura K, Nagai $H$, Akazawa M. Economic burden of community-acquired pneumonia among elderly patients: a Japanese perspective. Pneumonia. 2017;9:19.
3. Li G, Cook DJ, Thabane L. Risk factors for mortality in patients admitted to intensive care units with pneumonia. Respiratory Research. 2016;17:80.

4. Zec SL, Selmanovic K, Andrijic NL, Kadic A. Evaluation of drug treatment of bronchopneumonia at the pediatric clinic in sarajevo. Medical Archives. 2016;70(3):177-181.

5. Donà $\mathrm{D}$, Luise $\mathrm{D}$, Dalt $\mathrm{L}$, Giaquinto C. Treatment of community-acquired pneumonia: are all countries treating children in the same way?. International Journal of Pediatrics. 2017;20:4239268.

6. Watkins RR, Lemonovich TL. Diagnosis and management of community-acquired pneumonia in adults. American Family Physician Journal. 2011;83:1299-1306.

7. Mattila JT, Fine MJ, Limper AH, Murray PR, Chen BB, Lin PL. Pneumonia. treatment and diagnosis. Annals of the American Thoracic Society. 2014;11(Suppl 4):S189-S192.

8. Goldman, M.P dan Nair, R. .Antibacterial treatment strategies in hospitalized patients: What role for pharmacoeconomics? Cleveland Clinic Journal of Medicine. 2007;74(Suppl 
4):38-47.

9. Lu Z, Cheng Y, Tu X. Communityacquired pneumonia and survival of critically ill acute exacerbation of COPD patients in respiratory intensive care units. International Journal of Chronic Obstructive Pulmonary Disease. 2016;11:1867-1872.

10. Sarah JW, Shehzad A. Health outcomes in economic evaluation: the QALY and utilities. British Medical Bulletin. 2012;96(1):5-21.

11. Altaf M, Zubedi AM, Nazneen F. Costeffectiveness analysis of three different combinations of inhalers for severe and very severe chronic obstructive pulmonary disease patients at a tertiary care teaching hospital of South India. Perspectives in Clinical Research. 2015;6(3):150-158.

12. Negro RD, Eandi M, Pradelli L, Iannazzo S. Cost-effectiveness and healthcare budget impact in Italy of inhaled corticosteroids and brochodilators for severe and very severe COPD patients. International Journal of Chronic Obstructive Pulmonary Disease.
2007;2(2):169-176.

13. Saha L, Kaur S, Khosla P, Kumari S, Rani A. Pharmacoeconomic analysis of drugs used in the treatment of pneumonia in pediatric population in a tertiary care hospital in India: a cost-of-illness study. Medical Sciences. 2017;5(4):33.

14. Muller. Ceftazidime versus cefotaxime in the therapy of severe infections in intensive care patients. Infection. 2007; 15:173-178

15. Zhang S, Incardona B, Qazi SA. Costeffectiveness analysis of revised WHO guidelines for management of childhood pneumonia in 74 countries. Journal of Global Health. 2017;7(1):010409.

16. Huang SS, Johnson KM, Ray GT. Healthcare utilization and cost of pneumococcal disease in the United States. Vaccine. 2011;29:3398-3412. 